\title{
The Relationship of Changes in the Planet's Biosphere with the COVID-19 Pandemic and the Foundations of the Physical Theory of Virus Expansion
}

\author{
Evgeniy Shirokov \\ Department of Family Medicine, M. Sechenov First Moscow State Medical University, Moscow, Russia \\ Email address: \\ Evg-747747@yandex.ru
}

\section{To cite this article:}

Evgeniy Shirokov. The Relationship of Changes in the Planet's Biosphere with the COVID-19 Pandemic and the Foundations of the Physical Theory of Virus Expansion. International Journal of Clinical and Experimental Medical Sciences. Vol. 7, No. 4, 2021, pp. 74-80.

doi: $10.11648 /$ j.ijcems.20210704.11

Received: May 22, 2021; Accepted: June 7, 2021; Published: July 2, 2021

\begin{abstract}
In recent decades, epidemics of new, previously unknown human viral diseases, and their number is growing. The COVID-19 pandemic caused by the SARS-CoV-2 showed that the humanity is not prepared to deal with new challenges and does not understand the causes of aggression of viruses at all. An attempt to explain the catastrophic spread of the new coronavirus infection across countries and continents by the accidental transmission of the virus from animal to person is not really convincing. The pandemic was most likely caused by global changes in the planet's biosphere. According to V. I. Vernadsky, the biosphere consists of three main parts: plants, animals and microorganisms, with the constant total mass. In recent decades, the deforestation rate has increased dramatically. The number of wild animals has decreased as well, while the human population has increased. This growing imbalance has led to the expansion of viruses from animals to humans. The rapid spread of the infection in large ecosystems is associated with understudied physical properties of viruses. Viruses being a part of the nanoworld change the traditional ideas about the extension of infection per contiguitatem. There is every reason to believe that viruses have wave properties and are capable of creating stable resonance systems and an electromagnetic field. The energy of the electromagnetic field can be high enough to spread infection without any contacts and to fill the ecosystem with a new virus rapidly. Probably, in the external environment, viruses are able to create "coherent matter" - an electromagnetic field of high intensity, the energy of which is sufficient to convert into mass and, conversely, the mass of viruses into energy. New data on the wave properties of viruses explain the facts of the simultaneous occurrence and increase in the incidence of COVID-19 in different countries and populations. The expansion of viruses in ecosystems looks like a model of a complex oscillatory system. Such systems are capable of self-organization and demonstrate periodicity, which manifests itself in the form of waves of increasing and decreasing morbidity. The study of the ecological aspects of the pandemic and the physical properties of viruses leads to important practical conclusions: "new" viral infections are the result of global changes in the planet's biosphere, which lead to the expansion of viruses, the creation of new ecosystems. The development of these ecosystems is not only epidemiological, but also a complex physical process that requires further study.
\end{abstract}

Keywords: COVID-19 Pandemic, Wave Theory of Viruses, Expansion of Viruses

\section{Introduction}

In the mid-20th century, advances in epidemiology and hygiene, development of antibiotics and new diagnostic methods allowed to stop numerous epidemics of infectious diseases caused by pathogenic bacteria and protozoa. However, the early 21 st century showed that it was too early for the modern humanity to celebrate the thumping victory over pathogens. Epidemics of previously unknown, more and more severe viral diseases were even more frequent and aggressive. The COVID-19 pandemic caused by the SARS-CoV-2 showed that the humanity is not prepared to deal with new challenges. There was no system in place for predicting dangerous infections and a complete lack of understanding of the reasons for the growing expansion of viruses. A small extract from the final report of WHO experts, published on March 30, 2021, is 
sufficient proof of this statement [1]. Below are the main provisions of this report, with the wording similar to the original text. "When a new virus is discovered, it is important to understand its origin. It is very important to be able to identify and isolate the source and prevent the virus from further penetration into the human population. Numerous members of the coronavirus $(\mathrm{CoV})$ family are found in wild animals, which affords ground to classify them as zoonotic viruses. All SARS-CoV-2 strains obtained from humans to date are genetically closely related to coronaviruses isolated from bats." These assumptions are not sufficient to form a complete scientific understanding of the causes of the emergence and spread of new viral infections. Without questioning the wellknown facts about the biological properties of viruses, it is necessary to pay attention to the role of viruses in the biosphere of the planet, the ways of their interaction and selforganization. The study of the physical properties of viruses can significantly change the views of the scientific community on the nature of viral infections and lead to conclusions of great practical importance.

\section{COVID-19 Pandemics and the Biosphere of Earth}

Different scales of epidemic require appropriate tools to assess the situation objectively and consolidation of information at different levels. In order to localize an infection outbreak, identification of the pathogen and simple hygiene measures are enough. During an epidemic, quarantine and the significant administrative and medical resources must be used in order to prevent the uncontrolled spread of the disease. Studying the global changes in the planet's biosphere will allow to understand the causes and mechanisms of the pandemic.

There is an obvious discrepancy between the scale of the COVID-19 pandemic and the ideas of the scientific community about the reasons for the rapid spread of a new, understudied human infectious disease. The experts' point of view on the causes of the pandemic is particularly limited to the analysis of random scenarios that could lead to the transmission of a pathogenic virus from animal to human. While, 50 or 100 years ago human, contact with wild animals was closer, it did not lead to such large-scale zoonotic epidemics. The concept of a "new" viral infection also raises questions. This must be about "old" viruses that had a different habitat and were not studied by virologists and physicians. Hundreds of thousands of different viruses are always there in the biosphere, and only a few dozen of them are human pathogens. The species specificity of viruses has evolved for millions of years, and there is no reason for this order to be violated. In addition to the biosphere that changes significantly, which significantly disturbs the dynamic balance between the interacting organisms in the biocenosis. Why do these global changes occur? Without an answer to this key question, any response to the pandemic will not be sufficient. Vaccination will stop one pandemic, but the next "new" virus will make the world community face another, maybe even more significant danger. Lockdown will prevent the catastrophic result of the epidemic, but will not stop its spread.

Classical theory of V. I. Vernadsky suggests that the biosphere is a thin film of "living matter" that is directly involved in the continuous geochemical and geophysical transformations of the planet [2]. Biodynamically, the biosphere is a complex nonequilibrium oscillatory system, consisting of three main parts: plants, animals and microorganisms. The ratios of these parts, as well the numerous species and subspecies within these parts, determine the essence of interrelated and interdependent physical, chemical and biological processes that support life on the planet. Plants and animals are separated in space and localised, while members of the microworld (bacteria) and nanoworld (viruses) have no spatial restrictions in the biosphere (Figure 1). It takes hundreds of years for plants and animals to populate the entire planet surface. The environment favourable for the reproduction and conservation of plants and animals is limited by geophysical and climatic conditions. Bacteria, with their ability to multiply by fission exponentially, need only 4.5 minutes to colonize the entire planet [2]. How long it takes viruses to fill the biosphere is yet to be determined. The concept of mechanical space-filling is not applicable to viruses.

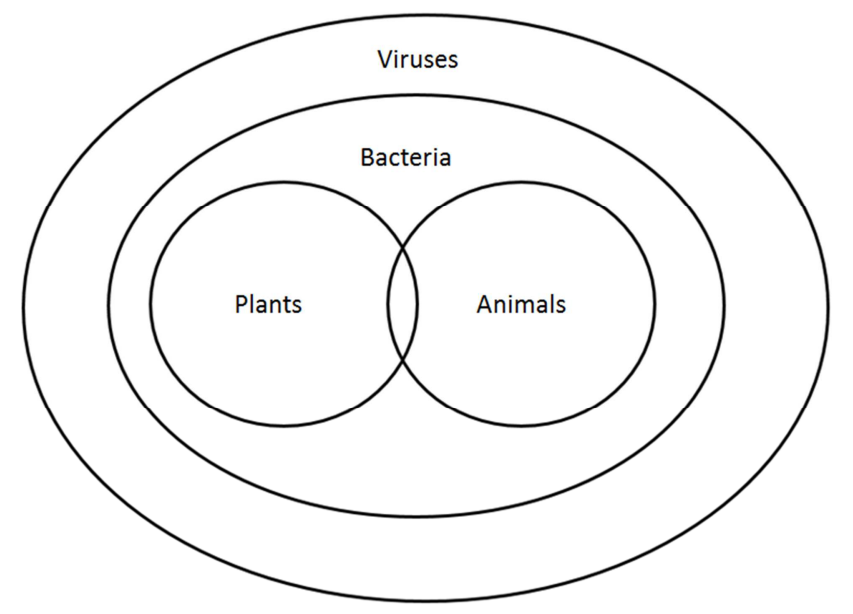

Figure 1. Spatial relationships between the main components of the biosphere.

Although the ratios of the biosphere components may change, the total mass of the biological matter is constant. Plants, animals and microorganisms interconnected in complex biochemical and biophysical metabolic processes ensure the stability and vitality of ecosystems [3]. The two largest ecosystems on the planet are the world ocean and land. Large ecosystems are territories with a fully formed biocenosis that are relatively homogeneous in terms of climatic, ethnic, economic and other characteristics. In the last 70-100 years, human activities have a significant impact on all land ecosystems - from countries and cities to continents. Agriculture, manufacturing, mining, energy, construction - all these activities create well-known environmental problems, which worsen every year. However, the viral expansion is likely related to deforestation. The deforestation rate on all continents has skyrocketed over the 
past 50 years, and continues to increase. From 2011 to 2015, $20 \mathrm{mln}$ hectares of forest were annually destroyed globally, since 2018 - $28 \mathrm{mln}$ hectares. The planet loses forest mostly due to meet the needs of manufacturing and construction, and to a lesser extent due to climate change and more frequent forest fires. In the last 40 years alone, the planet was deforestated on an area equal to that of the whole of Europe [4]. The dynamics of viral epidemics in recent decades roughly correlates with the deforestation rate.

Obviously, there is no direct link between logging and viral epidemics, but there is a chain of interrelated environmental problems that lead to significant imbalances, first in large ecosystems, then in the planet biosphere as a whole. Wild forests are the most complex ecosystems in which most species of animals, bacteria and viruses can reproduce and preserve. The destruction of forests causes an inevitable reduction in the number of animals in vast areas. Wild animals are a natural reservoir for many types of zoonotic viruses. A complex forest organism cannot be replaced by planting trees in city squares: it takes centuries to create a special forest biocenosis and the absence of men in this environment. A decrease in the proportion of plants and animals disturbs the dynamic balance in large ecosystems and causes the expansion of viruses outside the established habitat, including with a change in the species specificity that has evolved. A pandemic is the result of the destabilisation of the biosphere, which causes new ratios of the main components of the planet's biomass (plants, animals, microorganisms) to form. As the human population grows, and the number of wild animals decreases, biomass can be stabilised by employing a new vector - the expansion of microorganisms into the human component. In the world of microorganisms, there are probably the ratios of bacteria and viruses established as a result of evolution. The significant part of viruses is known to be bacteriophages. In ecosystems that humans have built for their lives, bacteria are more vulnerable than viruses. This is due to the massive use of antibiotics, which began in the late 20th century. This is not about medicine, where antibiotics are prescribed by doctors for specific indications in small doses. The problem is the industrial use of antibiotics in livestock and crop production. And we are talking not about milligrams, but thousands of tons. Thus, the new reality is a catastrophic deforestation, a decrease in the number of large and small wild animals, and anthropogenic pressure on bacterial communities. Viruses get a clear evolutionary advantage in their right to fill the biosphere. The growing human population becomes the natural environment for viruses. The concept of "expansion" seems to be more accurate than the concept of "epidemic" used to characterize this process. An epidemic is the result of infection with a pathogenic virus in a limited number of people and the subsequent spread of an infectious disease in the population. The expansion of viruses is the process of filling ecosystems with viruses that previously occupied another part of the biosphere. The viral expansion is not a contact-type spread of the epidemic (circles on the water), but a simultaneous increase in the proportion of new viruses in the biocenosis of biosystems that are distant from each other. Anti-SARS-CoV-2 were detected in Italians from March to September 2019 [5]. It is obvious that the expansion of SARS-CoV-2 occurred simultaneously in large, distant ecosystems long before the outbreak of the epidemic in China. The course of the epidemic process that could be observed on different continents (China - December 2019, Italy - March 2020, India - April 2021) demonstrates explosive dynamics that cannot be explained by contact-type transmission of pathogenic viruses from person to person. In addition to the well-studied infection routes, there must be other mechanisms for the spread of infection associated with the special properties of viruses.

\section{Physical Properties of Viruses}

Physics, as the most natural of natural sciences, studies the laws of nature.

Several facts can underlie important generalizations that can explain the reasons for the viral expansion and the unusual nature of the COVID-19 pandemic.

1) Modern biology cannot determine what exactly viruses are and establish whether they are living beings or not.

2) The assumption about the origin of viruses from a fragment of DNA that "escaped" a living cell is not convincing and exists only because there is no other more plausible assumption.

3) The nature of viruses as cellular parasites is also questionable.

4) If the use of the resources of a living cell for reproduction is considered as the main sign of parasitism, then humans using the resources of a living planet are the same as virus in this sense and are a planetary parasite. The principle of fractality makes these differences indistinguishable.

5) Science knows only a small number of viruses out of their endless variety.

6) The biochemistry and molecular biology of viruses have been studied in detail, but their physical properties and ways of existence outside their biological environment remain understudied.

7) The medical virological aspects have been more or less completely scientifically generalised.

8) Information about the geochemical role of viruses and their importance in the evolution of animals and plants is limited.

9) Very little is known about the informational and regulatory role of viruses in the planet biosphere, the interaction of viruses and viral communities with each other.

So, there are many unknown parameters in the equation that have resulted in a new viral pandemic. Empirical generalisations are needed to push the limits of the established paradigm, in which the parasite virus accidentally passes from animal to human and results in an epidemic that threatens the lives of millions of people. This paradigm suggests that there are only two solutions to the problem: limiting physical contact with animals and ill people, or 
vaccination to reduce susceptibility to the disease. Killing animals carrying a potentially dangerous virus is considered as an additional option. From 2019 to 2021, all three ideas were implemented to varying degrees in different countries, but this did not solve the problem. Obviously, without studying the nature (physics) of viruses, we will not be able to understand the causes of the pandemic.

Duality is the main difference between viruses and other living things that fill the biosphere. The duality of viruses is based on their affiliation with the microworld, where the particle size of a substance is measured in nanometers, mass is converted into energy, and vice versa. Viruses, with the sizes not exceeding the light wavelength, are more subject to the laws of Einstein's quantum physics rather than the Newton's mechanics. Viruses are capable of exhibiting the properties of a particle or a wave [6]. When the body is exposed to a virus, the virus acts as a toxic or infectious agent. Viruses can exist in a biologically active intracellular form and in the form of crystals - capsids [7]. Viruses are living matter and an important part of the biosphere. But viruses can exist outside the biosphere and then refer to nonliving matter. Viruses are extremely changeable, but stable in both biological and physical environments. The ability of viruses to change and adapt is incomparably higher than that of all known living creatures with slow evolution. Viruses in their number and diversity are many times superior to all life forms on Earth, but they cannot be detected by the senses, do not form a dense mass and do not occupy any volume in a limited space. The wave properties of viruses open up opportunities for them to spread quickly without the movement apparatus. The speed of viruses spreading in space can theoretically be close to the speed of light. This means that viruses are simultaneously present at the point where they are detected at the moment and at any other point in space [6]. Viruses interact with each other, fill ecosystems, taking part in the formation of biocenosis.

Table 1. Duality of viruses.

\begin{tabular}{ll}
\hline $\mathbf{X}$ & Y \\
\hline Particle & Wave \\
Toxic factor & Infectious agent \\
Living microorganism & Nonliving substance \\
Intracellular parasite & Carrier of genetic information \\
Dangerous to humans & Not dangerous to humans \\
Continuously changes & Resistant to any change \\
\hline
\end{tabular}

The duality of viruses is easier to understand and accept using the following linguistic key:

"Viruses are not only X, but also Y (not only Y, but also X)."

The ability of viruses to program cells of animals and plants indicates that the informational role of viruses in the biosphere is very important. From this point of view, the parasite-host relationship clearly does not reflect the role of viruses in the biosphere. The presence of viruses in the cells and tissues of the human body is not a synonym of a disease, but in most cases a fact of symbiosis, the meaning of which can go far beyond the concept of infection, persistence, carriage and asymptomatic course of the disease. For example, bacteriophage viruses are involved in the regulation of the number of bacteria in the human body. The obvious tendency of viruses to localise in the cells of the nervous system suggests the involvement of viral particles in the functioning of the neurohumoral regulation structures. The life of a multicellular organism from birth to death takes place in an environment filled with billions of viruses. Only a few dozen types of viruses are human pathogens. Others, being constantly present in space, pose no danger to humans whatsoever. But we do not know what the human environment would be without viruses. It is obvious that the interaction of viral communities creates a complex global network, with its principles of functioning yet to be studied.

\section{Interaction of Viruses}

There is very little information availabe about how viruses interact.

1) Within ecosystems and biological environments, viruses interact with each other, maintaining certain relationships between different species [8].

2) Ecosystems are filled with communities of viruses, which is not accidental, but regulated by the understudied selforganization of complex systems $[8,9]$.

3) Viruses do not emit any substances into the ambient environment, and the interaction of viruses cannot be mediated by biochemical reactions.

The issues of the interaction of viruses in large ecosystems, the sizes of which are incomparable with the size of viruses, remain understudied. It can be assumed that viruses communicate in space via electromagnetic waves — by radio (to put it simply).

Periodic oscillations of different frequencies, shapes and amplitudes are a property of living and nonliving matter [10, 11]. Elementary particles (an atom - a molecule — a substance - compounds of substances - cells and tissues living organisms) are subject to periodic oscillations relative to a fixed system of reference. The energy source for wave processes on a planetary scale is solar radiation, in the nanoworld - the energy of atomic bonds. Oscillations create electromagnetic waves, the energy of which in the most general terms is described by the well-known Einstein's equation: $\mathrm{E}=\mathrm{mc}^{2}$, and the relationship between the wavelength and its mass - by the de Broglie equation: $\lambda=\mathrm{h} / \mathrm{mv}(\mathrm{m}-$ the mass, $\mathrm{c}$ - the speed of light, $\mathrm{e}$ - the energy, $\lambda$ - wavelength, $\mathrm{h}$ - Plank's constant, v - speed). The intensity of electromagnetic radiation depends on the energy of the wave that passes through a certain area per unit of time.

The adaptation of mathematical terms to the ideas of biologists and physicians about viruses leads to unexpected conclusions and assumptions. The weight of the virus is so small that a single virus can be represented as an electromagnetic pulse with a wavelength less than that of light. G. P. Stupakov et al. found that the main viral human pathogens have their own stable frequency characteristics - a kind of wave passport. The specificity of the wave characteristics is determined by the shape and degree of symmetry of viruses [7]. By exposing a biological object infected with viruses to a weak electromagnetic field with 
variable characteristics, it is possible to detect the effect of resonant excitation, which indicates the presence of a virus and the intensity of infection (strength of the electromagnetic field) $[6,9]$. Obviously, resonant frequencies are found when the characteristics of the electromagnetic field of the virus community coincide with the diagnostic frequency. The energy flow density of the viral community can be quite high if the viral load is high, and the position of the viral particles in space is relatively stable. The assumption of a multiple increase in the electromagnetic field of viruses during massive infection is confirmed by experimental and theoretical studies [9-12]. The property of waves with similar frequency characteristics to coherently add up is well studied in quantum physics. Structurally homogeneous media achieve a uniform quantum state and behave like a large wave. A multiple increase in the energy of the electromagnetic field and its non-uniformity are the characteristic behaviour of wave systems. In the abmbient environment, viruses are likely to create "coherent matter" - a high-intensity electromagnetic field, with its energy sufficient to convert into mass and, vice versa, mass into energy [11]. Filling an ecosystem with crystal viruses can exhibit the properties of a maser, where many crystal elements create coherent radiation, and the spatial structure of uniform viruses acts as a resonator. The wave properties of viruses explain the non-uniform filling effect in ecosystems. Coherent matter in space creates areas of thickening (Bose-Einstein condensate) and vacuum zones low-energy areas (Figure 2).
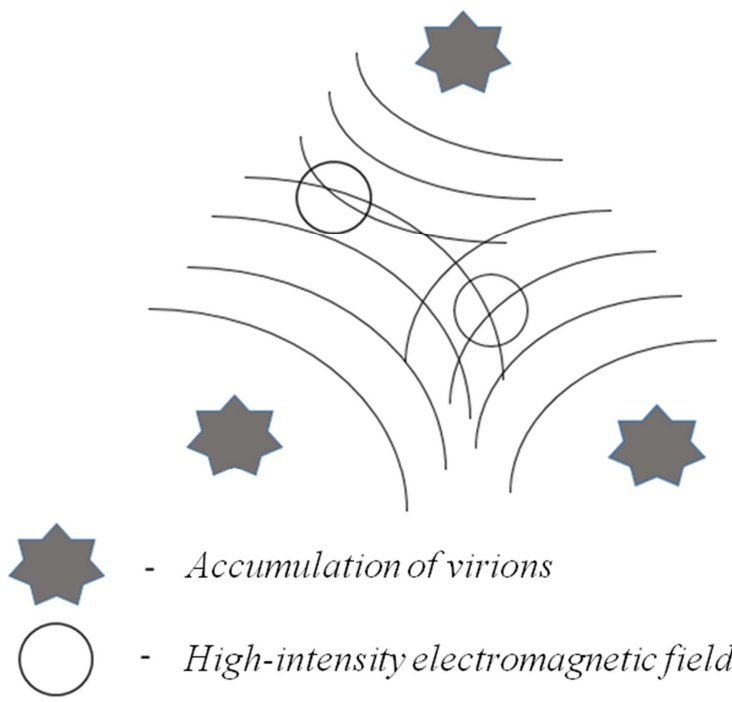

- Accumulation of virions

- High-intensity electromagnetic field

Figure 2. Non-uniform distribution of the intensity of the electromagnetic field.

Biologically inactive viral particles (virions) accumulated in space create stable resonance systems and a coherent electromagnetic field. The high-intensity electromagnetic field is capable of initiating the transition of viruses from an inactive form to a biologically active one. The mechanism of formation of an active virus from molecular subunits in a biological environment (self-assembly effect) was described by American virologist H. Frankel-Conrat in 1955 [13]. Self-assembly of a biologically active virus inside a cell influenced by a coherent electromagnetic field sounds quite unrealistically, but does not contradict the laws of quantum physics and synergetics. The mechanism of creating a biologically active virus from molecular subunits is likely to be associated with the selforganization of stable resonance systems [14]. The possibility of a virus (m) to be formed inside a biological system under the influence of the energy $(\mathrm{E})$ of coherent electromagnetic radiation fundamentally changes the idea of the spread of a viral infection. The energetic interaction of viruses determines the rate of filling ecosystems, the vector of spread of viruses and the ratio of species. Moreover, the intensity of the electromagnetic field of the viral condensate creates the possibility of non-contact transmission of the infection and allows us to make important practical assumptions:

1) During an epidemic, there can be high-energy virus zones and completely safe areas in a relatively small area.

2) Stable resonant viral systems are formed in a stationary environment, where physical conditions are created to enhance a coherent electromagnetic field.

3) The intensity of electromagnetic radiation is higher in an enclosed and confined space, where the energy flow is denser.

4) The dose of electromagnetic radiation depends on the exposure - the likelihood of infection is determined by the time a person stays in the condensate zone.

Filling ecosystems with a new virus looks nothing like filling space with a solid or liquid. Many viruses can exist in a confined three-dimensional space (cubic centimeter meter - kilometer) without taking up any volume. This phenomenon is more like filling the city with radio waves of different frequencies. There is reason to believe that the ratios of species in the biocenosis are regulated by the energy fields of viral communities. The expansion of new viruses disturbs the balance of the ecosystem. The high intensity of coherent electromagnetic radiation limits the further multiplication of viruses. Studies involving more than 44,000 cases of respiratory viral diseases have shown that upon reaching its peak activity, the spread of the viral pathogen of the disease stops [8]. There are complex synergistic and antagonistic competitive relationships between different types of respiratory viruses [8]. The dynamics of filling the ecosystem is also affected by the herd immunity that forms in the biological environment and localises the new virus. Two processes take place in the ecosystem and the population in parallel. Expansion and filling - in the ecosystem, illness in the population (Figure 3 ).

The new virus fills the ecosystem, creating an intense electromagnetic field, with its intensity limiting its further expansion. Filling is accompanied by an increase in illness frequency, which is limited by herd immunity and ecosystem filling. A new stationary state is achieved when the ecosystem is full, and all participants in the biocenosis reach their dynamic equilibrium. This means that the virus does not disappear as the illness frequency decreases. A stable ecosystem is resistant to new viruses, if the energy intensity is low. But an ecosystem that lost its equilibrium (unstable) is 
extremely sensitive to even a small amount of energy.

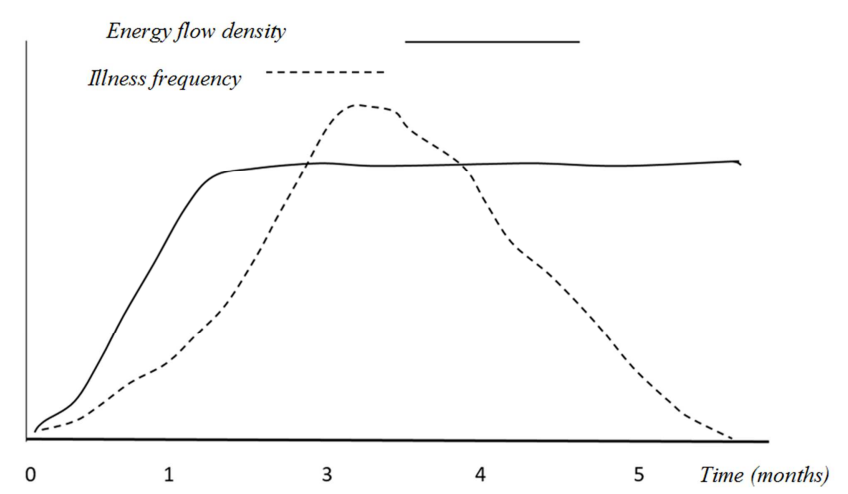

Figure 3. Filling the ecosystem with viruses and illness.

Therefore, the expansion of viruses in ecosystems looks like a model of a complex oscillatory system. Such systems are capable of self-organization and can achieve a stationary state - a relatively stable state that is resistant to destabilizing factors. However, dynamic systems that have lost their equilibrium react to "small stirrings" by transitioning to another, less stable state through the exacerbation mode [13-16]. The exacerbation mode of an unstable oscillatory system is characterized by loss and deformation of cycles, as well as an increased oscillation amplitude. As applied to the concepts of epidemiology, the exacerbation mode is actually an epidemic. The explosive growth in the incidence continues until the ecosystem is filled with new viruses and the transitioned to a stationary state with a different biocenosis.

\section{Environmental, Epidemiological and Clinical Generalisations}

COVID-19 pandemic:

1) For the first time in the past 100 years, it demonstrated a new quality of the epidemic process: the simultaneous involvement of large populations on different continents in the epidemic process.

2) It also showed that quarantine, as a universal response to epidemics of infectious diseases, is not effective.

3) It questioned the accidental transmission of the virus from animal to human.

4) It established a link between an infectious disease and global environmental problems.

In the new environmental conditions, viruses have got numerous advantages in filling ecosystems due to the growing imbalances in the main components of the biosphere. A multiple decrease in the number of wild animals and an increase in the human population over the past 200 years have created a new vector for the expansion of viruses. This vector is directed towards humans. The new reality is a natural increase in the likelihood of new viral infections in the near future. Understanding the essence of global changes in the biosphere will inevitably lead to solutions to environmental problems. First of all, it is necessary to stop the uncontrolled destruction of large forests, which can help to preserve numerous animal species. The creation and expansion of vast reserves can have a significant long-term impact on the emergence and spread of new viral infections.

The study of the physical properties of viruses explains some of the features of modern epidemics and opens up new directions in the fight against viral infections.

The wave theory of viruses assumes the existence of areas with high and low concentrations of viral particles, with high and low energy content of the environment. Infection depends on the "viral load", which is greater in areas with denser patient groups, in populated places where people are stable in space for a long time. The continuous movement of virus carriers prevents creating the necessary conditions for the formation of stable resonant systems. In moving masses of people, it is transmitted directly through physical contact, which cannot be prolonged, and personal protective equipment turns out to be quite effective.

Neighbouring ecosystems are filled with new viruses non-uniformly. The size of the ecosystem is important to achieve stabilisation. The energy of the viral electromagnetic field fades away in space in accordance with the laws of physics. The country with its administrative boundaries, climatic, genetic, ethnic and cultural characteristics is the optimal ecosystem for filling and achieving a stationary state. Theoretically, within a stable ecosystem, harsh restrictions are not needed. However, the movement of people from a stable ecosystem to an unstable one and vice versa leads to an exacerbation and an outbreak. The movement of large groups of people between large ecosystems is the main mechanism sustaining the pandemic. The cycle of filling a large ecosystem with a new virus lasts about 3 months. Repeated destabilisation may be associated with a new modified virus. It can be assumed that repeating waves of disease within the same ecosystem, in accordance with the laws of oscillatory system behaviour, will fade away. The cycle of such a system is as follows: destabilisation - exacerbation (filling) - stabilisation - a new stationary state. Isolation of large ecosystems (countries, continents) is the most effective way to limit the expansion of viruses. The period of complete isolation of ecosystems should probably be longer. The pandemic is changing the meaning of quarantine by increasing its scale. In a local epidemic, physical contact must be limited in a group of people or in a city. The pandemic increases the scale of restrictions fractally to countries or large geographic areas. No quarantine is needed within these ecosystems.

\section{Conclusion}

The study of the physical properties of viruses gives us an important practical conclusion: in addition to the traditional infection by airborne transmission, non-contact infection is possible. The expansion of viruses is the process of spreading in space viral particles capable of creating a non-uniform electromagnetic field. Even a short-term stay in the 
condensed energy area can lead to the formation of viruses inside the human body without direct transmission of an infectious agent from an ill person.

The development and rapid manufacturing of vaccines is the most affordable way to mitigate the problem of new viral infections. However, vaccination of large populations will always fail to keep pace with the epidemic by at least a year. The physical nature of viruses, the absence of typical signs of life in virions (respiration, metabolism) practically excludes traditional pharmacological methods of affecting the infectious process. But further study of the physics of viruses has a fairly high scientific potential. Physical limitation of the expansion of viruses does not look that wild.

\section{References}

[1] Origins of the SARS-CoV-2 virus. https://www.who.int/health-topics/coronavirus/origins-of-thevirus.

[2] Vernadsky V. I. Biosphere and noosphere / Preface R. K. Balandin. - M.: Airis-press, 2004. - 576 p.

[3] Taylor D., Green N., Soper R.. Biologycal science. Camdridge university press. 2004.

[4] Earth's forests are being cut down. And they are being cut down fast.

https://www.theworldcounts.com/challenges/planetearth/forests-and-deserts/rate-of-deforestation/story (Дата обращения 6.04.2021).

[5] Apolone1 G., Emanuele Montomoli E., Alessandro M., et al. Unexpected detection of SARS-CoV-2 antibodies in the prepandemic period in Italy. https://doi.org/10.1177/0300891620974755.

[6] Kantur V. A., Petrosjanc V. V. On the development of the wave theory of viruses. https://cyberleninka.ru/article/n/k-voprosurazrabotki-volnovoy-teorii-virusov.

[7] Kuznetsov Y., McPherson A. Atomic Force Microscopy in Imaging of Viruses and Virus-Infected Cells/ Microbiology and molecular biology reviews, June 2011, p. 268-285.

[8] Nickbakhsha S., Maira C., Matthewsc L. et al. Virus-virus interactions impact the population dynamics of influenza and the common cold. PNAS; 26: 52 (116): 27142-27150.

[9] Stupakov G. P., Semenov B. F., Sherbinina N. V., Rolik I. S. Patterns of development of chronic diseases and a new principle of their treatment: monograph - M.: Editorial and Publishing House of the Russian New University, 2021. -48 p.

[10] Herber R. Vibrational medicine. https://www.pdfdrive.com/vibrational-medicine-the-1handbook-of-subtle-energy-therapies-e162817922.html.

[11] Praschuk D. Coherent waves of matter. Chemistry and life. 2007; $3 . \quad$ https://elementy.ru/nauchnopopulyarnaya_biblioteka/430447/Khimiya_i_zhizn_3_2007.

[12] Prangishvili I. V., Birshtein B. I., Yaroshenko A. M. et al. Wave antiviral immunity. Theoretical justification. http://naukarus.com/volnovoy-antivirusnyy-immunitetteoreticheskoe-obosnovanie.

[13] Virology: textbook / A. V. Pinevich, A. K. Sirotkin, O. V. Gavrilova, A. A. Potekhin; edited by A. V. Pinevich. 2nd ed., add. - St. Petersburg: Publishing House of St. Petersburg. University, 2020.-442 p.

[14] Shirokov E. A. Hemodynamic crises. - M.: QUORUM Publishing House, 2011.

[15] Knyazeva E. N., Kurdyumov S. P. Fundamentals of Synergetics. Synergetic worldview. M.: KomKniga, 2005.-240 p.

[16] Arnold V. I. The theory of catastrophes. M.: Editorial UPSS, 2009. - $136 \mathrm{p}$. 